\title{
Cancer researchers get a grip on immune cell plasticity
}

\author{
Immune cells can transform themselves with plasticity that cancer labs seek to harness.
}

\author{
Vivien Marx
}

$\mathrm{n}$ $\mathrm{n}$ fantasy novels, people can readily turn into wolves and revert back. In real life, shape-shifting is common in immune cells, especially macrophages. These immune cells, sometimes called the innate immune system's 'first responders', can detect, engulf and destroy invaders. They can present some of an invader's tell-tale guts-antigens-to T cells and let those cells finish the destruction job. As tissue residents that tend to housekeeping, macrophages can also be more calmly vigilant. And they can follow a chemoattractant trail, invade and attack a tumor. But such tumor-associated macrophages (TAMs) can become bewitched.

Instead of finding and destroying cancer cells, TAMs can switch to promoting tumor cell growth and metastasis. This beguiling plasticity challenges cancer researchers. Understanding it is crucial to those who seek to deeply understand interactions between cancer and the immune system. Eventually, this could lead to ways of 're-educating' tumor-supportive macrophages.

When he first described TAMs ${ }^{1}$ in 1981 , his in vitro and in vivo evidence "ran against common wisdom," says Alberto Mantovani. He encountered skepticism due to the "rooted dogma" that immune cells always protected hosts, says Mantovani, who is scientific director of Istituto Clinico Humanitas in Milan and professor emeritus at Humanitas University.

The fact that macrophages can reside in various alternative phenotypic states is "by now, widely accepted, Mantovani having been a pioneer in this area," says Robert Weinberg, a cancer researcher at Whitehead Institute for Biomedical Research and who directs the MIT Ludwig Center for Molecular Oncology. Mantovani proposed M1 and M2 macrophages; the picture that has emerged is complex, with various intermediate states between M1 and M2. "Nonetheless, there is wide consensus that one type of macrophage is immunostimulatory and the other type is immunosuppressive," says Weinberg. How reversible the transition is, into and out of these alternative states, "is to my mind not settled."

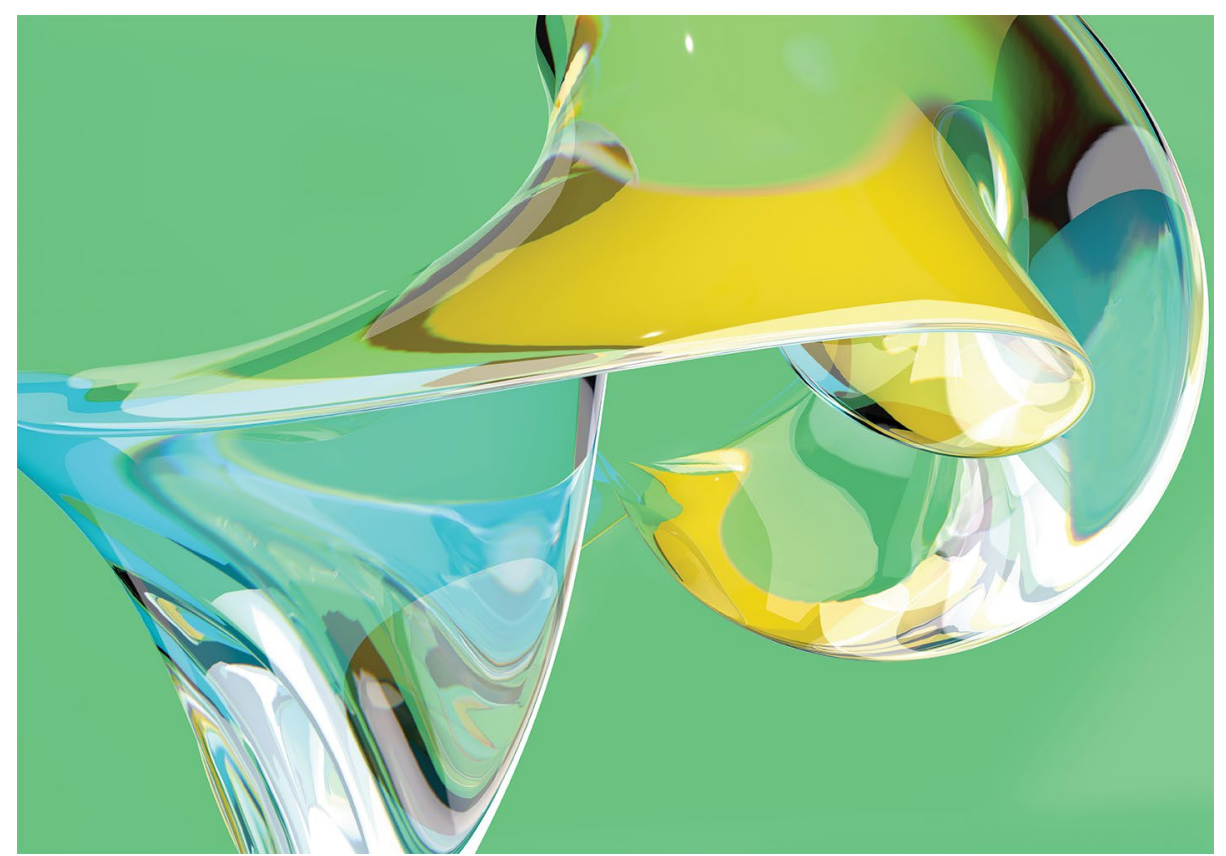

Macrophages show beguiling plasticity, which cancer labs work to understand by combining new and old methods. Credit: A. Onufriyenko / Getty Images

Plasticity is an underappreciated immunological trait, says Ido Amit, an immunologist at the Weizmann Institute of Science. Given the many pathways involved in plasticity, the M1/M2 divide is "so much over simplification that it doesn't really capture much of the truth," says Amit. To macrophages, it's Halloween every day: they have many costumes, he says. "They have this amazing ability to be plastic and respond." By digging into the details of when and how macrophages act as they do, immunologists want to find their role in driving cancer.

With checkpoint inhibitors, cancer's way of exhausting T cells can, in some cases, be successfully curtailed so the cells can attack tumors. Now, some labs and companies seek to leverage macrophage plasticity for treating cancer. Blood cells are formed in the bone marrow from stem cells. Blood cells include myeloid cells such as monocytes, which give rise to macrophages. Myeloid checkpoints may represent valuable therapeutic targets, on the basis of some early clinical trial results, says Mantovani. Engineered macrophages derived from induced pluripotent stem cells could be set up to attack tumors. Some approaches involve equipping macrophages with engineered receptors or outfitting them with tumor-toxic payloads. Companies include Carisma Therapeutics, Thunder Biotech, Myeloid Therapeutics and Bayer's BlueRock Therapeutics.

There are many unanswered basic research questions $\mathrm{s}^{2,3}$. "Plasticity is a hallmark of macrophages and represents a stumbling block for translation," says Mantovani. Single-cell analysis of TAMs and exploration of epigenetic mechanisms are revealing new aspects about macrophage diversity and plasticity.

Florent Ginhoux, a researcher in the Singapore Immunology Network at $A^{\star}$ STAR, observes this commercial activity, but his lab is not involved in such 
ventures. In his view, it's not yet clear how best to equip macrophages or direct their movement to a tumor.

Immunotherapy successes in cancer treatment based on the research of Nobel laureates Jim Allison or Tasuku Honjo show how powerful it can be to harness the immune system-in their case T cells-for eradicating a tumor, says Amit. Directing immune cells against cancer is a good plan. "I think that's definitely the future," and a future he's optimistic about. This will take time, especially given that macrophages "are a bit more difficult to understand because they are so plastic."

\section{Sensate cells}

Beyond macrophages that travel to a tumor, macrophages can be resident in tissues, where they engulf debris or pathogens ${ }^{4}$. Without resident macrophages, tissues would be like cities without garbage trucks, says Amit. When his lab assessed macrophages across tissue types, they found much diversity. The garbage truck macrophage that clears mucus in the lungs is unlike the one that breaks down worn out red blood cells in the spleen. The macrophages have traits in common, but "thousands of genes are different across these macrophages," says Amit. Other immune cells, such as dendritic cells, lack such diversity. A macrophage can sense its environment. "It's feeling where it is and adapting to that and changing much of its activity depending on where it is and what it has to do," he says.

Tumor tissue knows how to manipulate monocytes to, for example, become cells

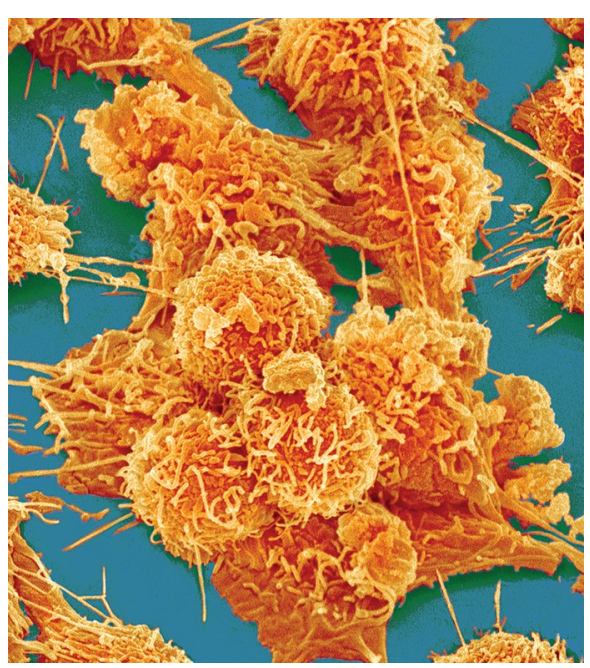

A tumor microenvironment such as the one modeled here can change immunosupportive macrophages into immunosuppressive, cancer-supporting ones.

Credit: Micro Discovery / Getty Images that inhibit the immune system, he says. Even when monocytes or macrophages are outfitted with chimeric antigen receptors (CARs), the environment can modify them. "In order to prevent that, you need to engineer them so they won't respond anymore naturally to the suppressive signals of the tumor," says Amit. That's why he is keen to use single-cell techniques to decipher the signals that pass between the immune system and tumors ${ }^{5}$.

One approach from his team is called PIC-seq ${ }^{6}$, a spatial technique that combines single-cell RNA-sequencing with analysis of doublets-cells that are locked in communication in a given tissue. The method, says Amit, is like taking two people conversing in a crowd and nudging them to the side as they continue talking. Fluorescent markers are used to detect and track the doublets through the workflow. The team worked out which enzymes were gentle enough to dissociate the tissue without separating these physically interacting cells.

Another method from his lab is INs-seq ${ }^{7}$, which integrates RNA-sequencing with a way of assessing intracellular proteins, signaling and metabolic pathways. The team uses it to keep an eye on TREM2 (triggering receptor expressed on myeloid cells 2 ). This gene encodes a protein on the surface of macrophages. It helps activate cells involved in obesity, and it's involved in progression to metabolic disease. TREM2 also appears to be involved in immunosuppression in the tumor microenvironment. TREM2 marks immunosuppressive myeloid cells and thus helps researchers to untangle signaling between tumors and immune cells.

\section{Beyond flow cytometry}

From the start of research in this area, functional analysis was used to distinguish differentially polarized macrophages, says Paola Allavena. She is group leader of cellular immunology at the clinical and research institute of Humanitas. Such analysis includes looking at how cytotoxic macrophages are toward tumor cells and teasing out secreted mediators, such as immunosuppressive or immunostimulatory cytokines, she says. "Flow cytometry is probably the most popular method to profile macrophages," says Allavena, one that has advanced greatly in the last two decades. It has led the way to, for example, multiparametric analyses. Flow cytometry, however, relies on the availability of reagents-antibodies-that target identified surface molecules. In more recent years, transcriptomic analysis and single-cell RNA studies have delivered a more global type of information on macrophages, including TAMs, she says, and yielded "a picture of

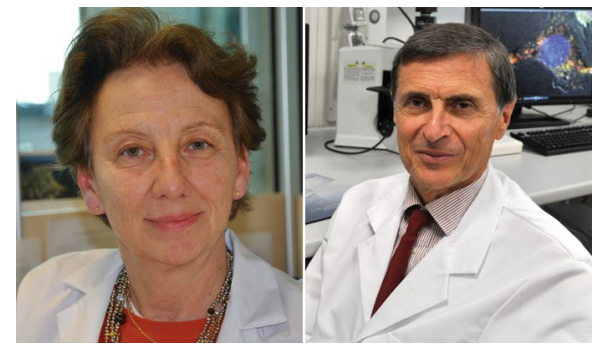

Paola Allavena and Alberto Mantovani have long been studying the role of macrophage plasticity in cancer. Credit: Humanitas Institute

the heterogeneous macrophage world that is much more complex than previously perceived."

Mantovani, Federica Marchesi and colleagues recently performed single-cell analysis of TAMs in human liver metastases from colorectal cancer-to their knowledge, the first single-cell analysis of myeloid cells in human metastases ${ }^{8}$. They sought to differentiate immunosuppressive from immunosupportive ones and distinguish them from the macrophages resident in healthy liver that support tissue homeostasis. Classic immunohistology methods revealed how heterogeneous the macrophages were in terms of shape and size. Some macrophages in the tumor samples, apparent with CD68 staining, were small and round; others were spiky and elongated, and there were many shapes in between. M1-like macrophages tend to be rounder and flattened whereas M2-like cells are more elongated.

The scientists quantified this diversity using RNA sequencing and comparing metabolic pathways, performing immunohistochemistry for the M2-like marker CD163, and using flow cytometry for general cell sorting. They assessed the large and small macrophages from 5 patients, later expanded to 101. Larger TAMs (L-TAMs) had more intracellular complexity, such as vacuoles, and they found over 1,100 genes differentially expressed genes in L-TAMs, which had an upregulated liver X receptor (LXR) pathway-a lipid metabolism pathway-compared to small TAMs (S-TAMs).

Discerning different types of macrophages is about characterizing their different states. For example, says Allavena, macrophages can be stimulated with interferon- $\gamma$ to become more similar to M1-like TAMs and signals such as interleukin-4 can nudge them toward an M2-like identity. The scientists developed a machine-learning approach for their quantitative assessment and found the clinical course of cancer was strongly correlated to macrophage morphology 


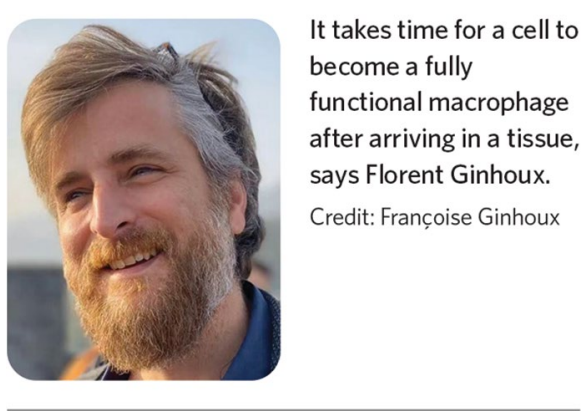

and transcriptional profile down to the single-cell level.

For A*STAR's Ginhoux, macrophage research is empowered by techniques such as mass cytometry, which applies antibodies tagged with heavy metal isotopes and single-cell RNA sequencing. Such techniques have led to "an explosion of descriptions of clusters of populations of cells with different expression profiles and more and more markers," he says. This holds true for both macrophages in cancer and their "steady state" as resident macrophages in tissues. "And I think people are more and more puzzled by the level of heterogeneity of immune cells and especially of macrophages," he says.

M1 and M2 have been "a very important and interesting concept," but this dichotomy is changing as technology emerges to characterize cells in ever more ways. It's becoming accepted that it's rather a "continuous spectrum of differentiation" bookended by M1 and M2. It's not a line but more of a polygon with plenty of in-between states. Some macrophages express certain cytokines and not others, and phenotypes vary along a spectrum from inflammatory to supportive. With flow cytometry experiments, one can gate for macrophages, some of which might have been resident in a tissue and others of which might have developed from monocytes. He likes using several techniques and integrating data. His training taught him to define cells by "CDs 1 to whatever," and he has added to his methods: mass cytometry with a CyTOF system from Fluidigm, multiplexed spectral flow cytometry with a Cytek instrument that enables his team to use around 30 markers, single-cell RNA sequencing, high-dimensional protein profiling and epigenetic analysis, too.

But time is not readily captured with these techniques. Among the diversity of thousands of cells are some just-produced macrophages while others are experienced in signaling with other cells, says Ginhoux. Timing matters, for example, in brain development. As Amit says, microphages must enter the brain at a particular time during development to develop adequately as microglia.

"People think that when cells arrive in a tissue, magically they become macrophages in a few days," says Ginhoux, but it takes time, perhaps months, to become a fully functional macrophage. Signaling and epigenetic events occur in a given niche. When cancer occurs, a niche is shaken up and a new microenvironment is established. Macrophages can become specialized on the basis of their tissue of residence. Although this is known, says Ginhoux, what's insufficiently heeded is how it affects the macrophages. A macrophage in the lung receives different signals from its neighbors than a macrophage in the liver. This leads to plasticity and heterogeneity. One important methodological frontier he sees is ways to track and characterize such traits and to capture sub-tissue spatial localization of macrophages, such as for comparing those residing near blood vessels with those close to nerve bundles. Such approaches will help with teasing out what happens in healthy tissue and in cancer.

\section{Mice and models}

Together with colleagues at Shanghai Jiao Tong University and the University of Bonn in Germany, Ginhoux and his team built fate-mapping models to distinguish macrophages by origin ${ }^{9}$. It was long believed that all macrophages derive from circulating monocytes. Says Ginhoux, that's true for some macrophages, which are continuously replaced and replenished. Other macrophages, though, originate during embryonic development.

With the team's mouse models, the scientists can track and time-stamp monocytes and macrophages. To do so, they generated fate-mapping mice with fluorescent reporters inserted into the $M s 4 a 3$ gene, a gene expressed by monocyte precursor cells. This lets the researchers specifically trace monocytes and granulocytes and quantify how many monocytes become tissue-resident macrophages in heathy tissue and in inflammation. They also made a mouse with an inducible gene to track monocyte-derived cells. "We can track now the time issue: are you a young or an old monocyte-derived cell?" he says.

The team is using these mice in their cancer research projects. "My lab is investing a lot, a lot of time into cancer and timestamping," says Ginhoux. They have shared the mice with around 30 labs, but sharing has been slowed by the pandemic.

As the experiments progress, "we are discovering more and more complexity," he says. When a tumor starts, most of the macrophages are tissue-resident macrophages, mainly embryonic types, but are they are slowly replaced by monocytes. This is the first layer of complexity.

As the tumor grows, inflammation ensues. Monocytes are recruited and TAMs become numerous. T cells, too, are recruited and are subverted by the tumor microenvironment, turning immunosuppressive. Many studies indicate the more macrophages in a tumor the worse the prognosis, says Ginhoux. Monocytes and macrophages may be shifting their states upon arrival at the tumor as a result of the signals they experience. But messages travel and the signals may be even sensed in the bone marrow where monocytes are made. "Maybe the monocytes might be recruited even already compromised," he says.

As Xingtong Liu at The Jackson Laboratory (JAX) points out, when labs select immunodeficient mouse models for studies such as those on the immune system or cancer, they will consider the fact that inbred lines show more consistent tumor growth whereas tumor growth in outbred mice will be more varied due the animals' genetic heterogeneity.

The murine immune system can prevent human tumors from engrafting, which is why labs use immunocompromised strains. JAX has a series of them. Humanized NSG mice, based on the NOD scid- $\gamma$ mouse developed at JAX, support engraftment of human hematopoietic stem cells and human peripheral blood mononuclear cells. These can be used to study human T cell immunomodulation. There is a NSG-SGM3 strain that supports human myeloid and regulatory $\mathrm{T}$ cell development. The $\mathrm{CD} 34^{+}$ HSC NSG-IL15 strain develops mature

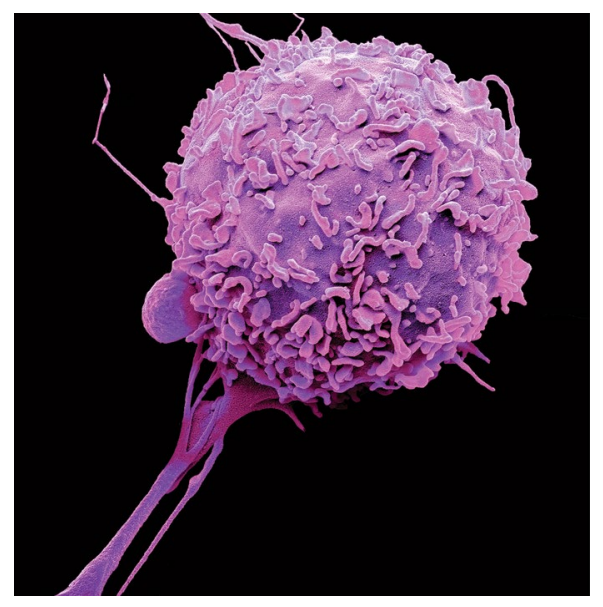

Some macrophages travel to a tumor, but there are also macrophages resident in tissues, where they engulf debris or pathogens. Credit: Steve Gschmeissner/Science Photo Library 


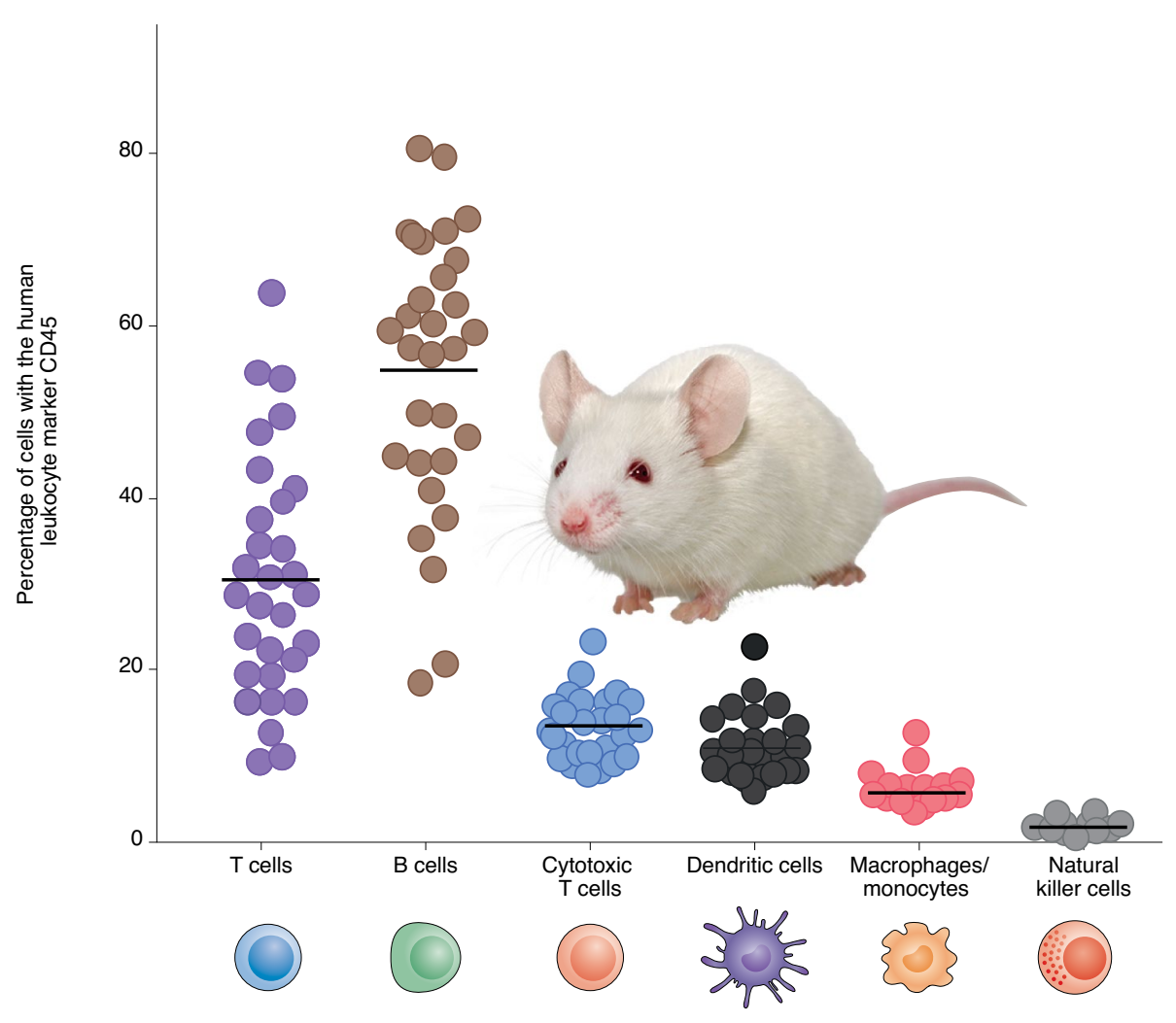

Mouse models help labs study the interaction between a tumor and the immune system. Mice like Taconic's HuNOG-EXL mouse are made to express traits of the human immune system. Credit: Taconic Biosciences; Thomas Phillips, Springer Nature

human natural killer cells at levels found in people.

Advanced humanized mice are ways to model dynamic events in the immune system and plasticity of macrophages. But no model recapitulates all human immunity, says Paul Volden, a cancer biologist at Taconic Biosciences who helps labs find models for their experimental questions. The more humanized a mouse, the more complexity there is.

A human immune system is geared toward recognizing "self versus non-self." The human cells in the mouse can see all the tissue around themselves as foreign and "will mount an immune response and attack the host and kill it," he says. Model developers have to set up one "that walks the line of human and useful versus too human and attacking the host." Mouse models are challenging to make, but they're needed given the growth and successes in immuno-oncology. "It's moved so fast," he says. Over the last few years, he has observed a shift from approaches focused on T cells to questions asked of other immune cells. "The largest interest is in the myeloid cell space," he says.

Researchers might look for human natural killer cells or certain types of human macrophages. For some experiments, they might need those macrophages or natural killer cells to be localized to a xenograft human tumor. "Then the question becomes, are they there?"

At a 2018 cancer conference, Volden saw a poster by a team of scientists at Tesaro, a biotech that GlaxoSmithKline acquired in 2019. Tesaro was looking at a way to improve $\mathrm{T}$ cell therapy with a drug candidate that works on cells in the myeloid lineage. The study involved a programmed cell death protein-1 (PD-1) inhibitor and a compound that targets lymphocyte activation gene-3 (LAG-3).

The combination enhanced $\mathrm{T}$ cell function, amplified the effect of the PD-1 inhibitor and reduced the number of immunosuppressive TAMs more than the PD-1 inhibitor alone. "Not only do you get macrophages in there," he says, "you can perform full analyses within the tumor." This model makes it possible to characterize TAMs as well as M1-like and M2-like macrophages in sufficient numbers for flow cytometry. The Tesaro work was, to his knowledge, the first dataset in which humanized TAMs infiltrated a tumor in the new mouse Taconic had developed.

The team had used Taconic's HuNOG-EXL mouse, which is "super-immunodeficient," to enhance engrafting human tumors, says Volden. The mouse expresses human cytokines such as human granulocyte-macrophage colony-stimulating factor (GM-CSF) and human interleukin-3 cytokines that are brought into the mice with $\mathrm{CD} 34^{+}$human hematopoietic stem cells. The animals have cells of the human myeloid and lymphoid lineages, including macrophages, not only in their peripheral blood but also in non-lymphoid organs such as lung and liver.

\section{Plastic and on fire}

The polarization of macrophages toward the pro-tumor M2-like state or antitumor M1-like states can directly affect the anti-tumor functions of T cells, says Anushka Dongre, a postdoctoral fellow in the Weinberg lab. For example, M1-like or antitumor macrophages promote the expansion of $\mathrm{T}$ helper type $1\left(\mathrm{~T}_{\mathrm{H}} 1\right)$-like $\mathrm{CD}^{+} \mathrm{T}$ cells and CD8 ${ }^{+} \mathrm{T}$ cells. Both of these cell types have known antitumor functionality. By contrast, M2-like macrophages secrete, among many factors, arginase, which can deplete arginine reserves from the tumor microenvironment, she says. That, in turn, can dampen the proliferative capacity and cytolytic functions of T cells. "So not only is the phenotypic plasticity of macrophages widely accepted, but it also has direct consequences on modulating the antitumor activity of T cells," says Dongre.

That macrophages are involved in tumor-promoting inflammation "is now accepted by the community," says Mantovani. In his view, inflammation is a hallmark of cancer.

Indeed, says Weinberg, "inflammation is now widely acknowledged as a predisposing condition for the formation of a broad array of different types of human tumors." Inflammation within a tissue creates a fertile microenvironment for the inception of cancers. That raises the question of whether, once cancer cells are formed, inflammatory traits are retained and continue to accompany the behavior of these cells. Here, he says, the "the answer is likely to be yes," especially in the context of the epithelialmesenchymal plasticity and the role of the cell-biological program termed the epithelial-mesenchymal transition (EMT), "which undergoes activation in highly progressed carcinomas as these cells transit increasingly toward a quasi-mesenchymal phenotypic state that includes a number of markers of inflammation."

Macrophages are not alone in their penchant for phenotypic plasticity. Work in the Weinberg lab has, for example, shown that cells committed to an epithelial differentiation state may thereafter, in 
response to contextual signals, move reversibly into more mesenchymal states and may actually move back and forth between various alternative phenotypic states. Even after commitment to a major differentiation lineage, says Weinberg, macrophages and a variety of other cells in the body can move from one phenotypic sub-state to another, and do so in response to various types of contextual signals they receive. "Indeed," he says, "this phenotypic plasticity is likely to be the rule rather than the rare exception."

\section{Vivien Marx ${ }^{凶}$}

Nature Methods.

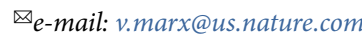

Published online: 26 March 2021

https://doi.org/10.1038/s41592-021-01112-8
References

1. Mantovani, A. Int. J. Cancer 27, 221-228 (1981).

2. Cassetta, L. \& Pollard, J. W. Nat. Rev. Drug Discov. 17, 887-904 (2018).

3. Kuo, C. J. et al. Cancer Cell 38, 145-147 (2020).

4. Guilliams, M. \& Svedberg, F. R. Nat. Immunol. 22, 118-127 (2021).

5. Yofe, I., Dahan, R. \& Amit, I. Nat. Med. 26, 171-177 (2020)

6. Giladi, A. et al. Nat. Biotechnol. 38, 629-637 (2020).

7. Katzenelenbogen, Y. et al. Cell 182, 872-885.e19 (2020).

8. Donadon, M. et al. J. Exp. Med. 217, e20191847 (2020).

9. Liu, Z. et al. Cell 178, 1509-1525 (2019). 